\title{
Feasibility of Using EMG for Early Detection of the Facial Nerve During Robotic Direct Cochlear Access
}

\author{
*Juan Ansó, †Christina Stahl, *Nicolas Gerber, *Tom Williamson, \\ *Kate Gavaghan, †Kai Michael Rösler, §Marco-Domenico Caversaccio, \\ *Stefan Weber, and *Brett Bell \\ *ARTORG Center for Biomedical Engineering, $\dagger$ VetSuisse Faculty, $\ddagger$ Department of Neurology, ENMG-Station \\ and §Department of ENT Head and Neck Surgery, Inselspital, University of Bern, Bern, Switzerland
}

\begin{abstract}
Hypothesis: Facial nerve monitoring can be used synchronous with a high-precision robotic tool as a functional warning to prevent of a collision of the drill bit with the facial nerve during direct cochlear access (DCA).

Background: Minimally invasive direct cochlear access (DCA) aims to eliminate the need for a mastoidectomy by drilling a small tunnel through the facial recess to the cochlea with the aid of stereotactic tool guidance. Because the procedure is performed in a blind manner, structures such as the facial nerve are at risk. Neuromonitoring is a commonly used tool to help surgeons identify the facial nerve (FN) during routine surgical procedures in the mastoid. Recently, neuromonitoring technology was integrated into a commercially available drill system enabling real-time monitoring of the FN. The objective of this study was to determine if this drilling system could be used to warn of an impending collision with the FN during robot-assisted DCA.

Materials and Methods: The sheep was chosen as a suitable model for this study because of its similarity to the human ear anatomy. The same surgical workflow applicable to human patients was performed in the animal model. Bone screws, serving as reference fiducials, were placed in the skull near the ear canal. The sheep head was imaged using a computed tomographic scanner and segmentation of FN, mastoid, and other relevant structures as well
\end{abstract}

as planning of drilling trajectories was carried out using a dedicated software tool. During the actual procedure, a surgical drill system was connected to a nerve monitor and guided by a custom built robot system. As the planned trajectories were drilled, stimulation and EMG response signals were recorded. A postoperative analysis was achieved after each surgery to determine the actual drilled positions.

Results: Using the calibrated pose synchronized with the EMG signals, the precise relationship between distance to $\mathrm{FN}$ and EMG with 3 different stimulation intensities could be determined for 11 different tunnels drilled in 3 different subjects.

Conclusion: From the results, it was determined that the current implementation of the neuromonitoring system lacks sensitivity and repeatability necessary to be used as a warning device in robotic DCA. We hypothesize that this is primarily because of the stimulation pattern achieved using a noninsulated drill as a stimulating probe. Further work is necessary to determine whether specific changes to the design can improve the sensitivity and specificity. Key Words: Cochlear implant—Facial nerveMastoidectomy-Neuromonitor.

Otol Neurotol 35:545-554, 2014.
Today, cochlear implantation procedures are performed much the same as when the implants were first introduced more than 3 decades ago, namely, through a mastoidectomy. Several research efforts have sought to reduce the invasiveness of the implantation procedure by avoiding the

Address correspondence and reprint requests to Brett Bell, Ph.D., ARTORG Center for Biomedical Engineering, University of Bern, Murtenstrasse 50, 3010 Bern, Switzerland; Tel: 004131632 7589; Fax: 004131632 7624; Email: brett.bell@artorg.unibe.ch

Conflicts of Interest and Source of Funding: The authors received funding from the Swiss National Science Foundation (Co-Me) and MedEl GmbH (Innsbruck, Austria) for work related to the accuracy. The authors received the EMG monitoring equipment and consumables from MEDTRONIC AG (Switzerland), and surgical equipment and consumables from Bien-Air Medical Technologies (Switzerland). mastoidectomy $(1,2)$, but questions of safety and other complications, such as electrode extrusion and undesirable insertion angles, have limited the acceptance of these alternative procedures. Alternatively, a procedure termed direct cochlear access (DCA) avoids an open mastoidectomy by relying on stereotactic tool guidance to accurately and reproducibly drill a small diameter tunnel to the middle ear cavity. Free hand stereotactic guidance $(3,4)$, patientspecific templates $(5,6)$, and robotic systems $(7,8)$ have been used to guide a drilling tool to a target location on the cochlea. Regardless of the stereotaxic means, each of these methods suffers from the fact that they rely entirely on the navigation system to ensure that the procedure is carried out as planned. Therefore, additional safety measures 
independent of the primary tracking system are required to detect possible system error and ensure most importantly the preservation of the facial nerve (FN).

Currently, electromyography (EMG) based neuromonitoring (NM) is commonly used to aid the surgeon in locating the FN during common otologic procedures (9-12). Although the use of FNM has been advocated by many, it is not universally applied, nor is there a consensus about its benefits in middle ear surgery $(13,14)$. Despite this lack of agreement, it is accepted that FNM can help the surgeon to identify the $\mathrm{FN}$ in revision, malformation, cholesteatoma, and cochlear implantation cases $(15,16)$. The recent integration of this monitoring technology into a surgical drill (Visao StimBur, Medtronic, USA) presents an opportunity for additional safety during DCA procedures. However, the sensitivity and specificity of FN detection by the integrated NM system must be established.

Previously, Bernardeschi et al. (17) attempted to determine a correlation between distance to the FN and stimulation intensity during a translabyrinthine approach for vestibular schwannoma removal. Measurements were obtained by stopping the milling process at the first warning from the NM system and subsequent visual inspection of the remaining fallopian canal wall bone thickness in postoperative CT images. In that study, the authors were able to show that the drill-integrated NM system elicited an EMG response with a remaining bone thickness of 0 to $2.8 \mathrm{~mm}$ using a $1.0-\mathrm{mA}$ stimulation current. To our knowledge, this represents the only study to date where an estimate of the distance to the facial nerve was obtained using a drillintegrated NM system.

Within this work, we present an integration of the StimBur (Medtronic, USA) NM device with a previously described imaged-guided surgical robot, which was recently shown to have a drilling accuracy of $0.15 \pm$ $0.08 \mathrm{~mm}$ (18). In contrast to the open cavity created in traditional procedures in the mastoid, the minimally invasive robotic approach results in a significantly different electrical scenario (tunnel drilling versus cavity milling). Furthermore, the robot system can provide a more precise estimate of the tool-tip position relative to the anatomy, thus providing a better measure of the distance/stimulation relationship. If a repeatable correlation exists, this could be used to provide an early warning of FN collision during DCA. The feasibility of the approach was evaluated in an animal experiment where several tunnels were drilled at differing distances from the $\mathrm{FN}$ using the surgical robot system.

\section{MATERIALS AND METHODS}

\section{Robotic Surgical Navigation System}

The robotic surgical navigation system was developed specifically for surgical interventions on the lateral skull base with a focus on cochlear implantation (19). The main component of the system is a light-weight robot arm, which can be easily mounted on the side rails of a standard OR table. The size and configuration of this arm allow it to move tools (surgical drill) in and out of the surgical zone as needed, whereas the base remains stationary on the side rail mount. The system also includes custom interactive planning (OtoPlan) and navigation (OtoNav) software modules. The planning module uses $3 \mathrm{D}$ diagnostic image data to accurately locate (segment) important anatomic structures (facial nerve, external auditory canal, cochlea, etc.). Using these segmented structures, a safe and effective drill trajectory is then defined. This plan is accurately referenced by the robot system by matching bone anchored screw locations in the physical or patient space with the same points in the image space as is reported in (20). Accurate reproduction of this plan is enabled through precise robotic positioning and accurate referencing of the tool location relative to the patient using a high-accuracy optical tracking system (CamBar B1, Axios $\mathrm{GmbH}$, Germany).

\section{NM Integration and Setup}

An otologic drill (Visao, Medtronic, USA) with integrated facial nerve monitoring attachment (StimBur, Medtronic, USA) was fixed to the robot end effector through a special adapter. An optical tracking reference was also attached and calibrated relative to the drill axis. The other main components of the NM system include the power control unit (IPC, Medtronic, USA) and the stimulation and monitoring unit (NIM 3.0, Medtronic, USA). A data interface (DAQ-NI 6215, National Instruments, USA) between the stimulation monitor and the navigation system was also implemented to enable exchange of stimulation/ feedback signals as well as the setting of stimulation intensity during a given test. An overview of these components as configured in the OR is shown graphically in Figure 1.

The NIM 3.0 was set with the following parameters. The stimulation pulse-width and frequency was set to $100 \mu \mathrm{s}$ and $4 \mathrm{~Hz}$, respectively. EMG signals were recorded in 2 channels continuously. Synchronization with the electrical stimulus events was performed to enable proper identification of electrically elicited EMG responses. The stimulation period was divided into 3 subsegments. Segment $1(3.1 \mathrm{~ms})$ was defined as the rejection period wherein the stimulation artifact is removed. Segment 2, starting at $3.1 \mathrm{~ms}$ and ending at $50 \mathrm{~ms}$, was used as a sampling window for electrically elicited responses. EMG signals arising during the final $200 \mathrm{~ms}$ (Segment 3) were attributed to other stimuli such as mechanical trauma or high temperatures.

Using the input from the data interface, the stimulation intensity can be automatically modulated by the navigation software. Using this method, stimulation current was increased from 1.5 to $2.5 \mathrm{~mA}(0.5-\mathrm{mA}$ increment) and back again within a period of 1 second during the drilling phase. Analogue EMG signals from the NIM 3.0 were digitized with a 16-bit resolution and a 40 ksamples $\cdot \mathrm{s}^{-1}$ sampling rate with no additional analogue filtering. EMG signals were recorded synchronously with the tool position relative to the planned trajectories (correspondence achieved through registration process).

\section{Animal Model}

Sheep was chosen as an appropriate model because of the similarities between the middle and inner ear anatomy with humans. Additionally, the sheep ear has been described as an appropriate model for otologic surgery and training (21). Morphometric studies of the middle and inner ear revealed that the ear structures are generally similar in sheep, but two-thirds the size of those in humans (22). The main differences identified were the presence of an air-filled bulla tympanica and the absence of air cells in the mastoid in sheep (23).

Four surgeries on sheep were conducted with approval of the local institutional review board (Bernese cantonal animal 


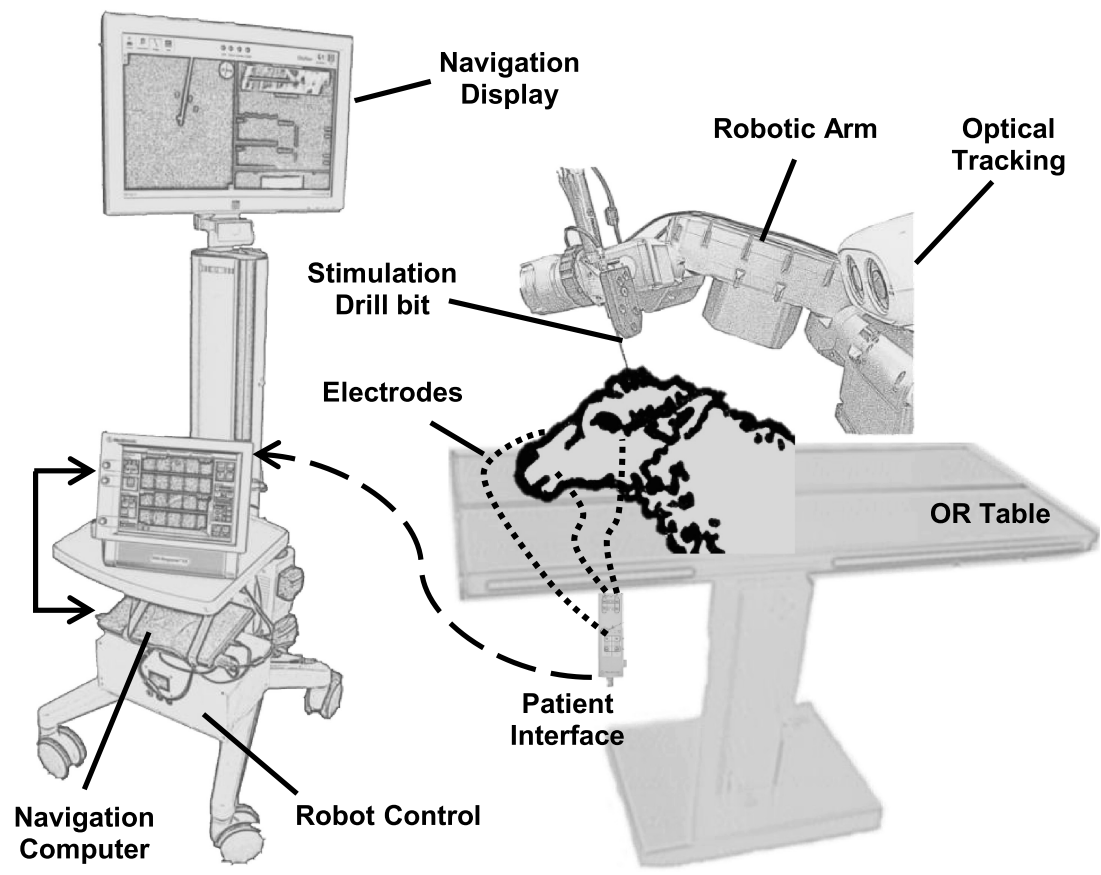

FIG. 1. Overall experimental setup consisting of the animal, electrodes, and system setup.

commission, approval number 56/12). Animals were first desensitized with $0.1 \mathrm{mg} / \mathrm{kg}$ diazepam and $0.1 \mathrm{mg} / \mathrm{kg}$ butorphanol administered intravenously. General anesthesia was induced with an intravenous injection of thiopental $2.5 \%$. Endotracheal intubation was performed whereby the anesthetic state was maintained by isoflurane in $100 \%$ oxygen. Ringers lactate solution was administered at a rate of $10 \mathrm{ml} / \mathrm{kg}$ per hour. Standard anesthesia monitoring included the following: ECG, pulse oximetry, capnography, inspiratory and expiratory inhalant anesthetic concentrations, and invasive blood pressure. To ensure normal facial nerve activity, no neuromuscular blockade was used in the anesthetic protocol.

\section{Imaging and Planning}

After anesthetic induction, surgical access to the mastoid region was gained by excising the temporalis muscle. Next, 4 small $\varnothing 1.5 \times 3 \mathrm{~mm}$ titanium screws (M-5220.03, Medartis, Switzerland) were implanted near the auditory canal for later referencing of the physical and image spaces. Subsequently, the subject head was imaged with a $0.75 \times 0.5 \times 0.5 \mathrm{~mm}^{3}$ voxel size using a 16-slice CT scanner (Brilliance, Philips AG). Planning of the procedure was performed using a custom software tool (24). First, the positions of the 4 implanted screws are found using a model-based matching method (20). Segmentation of the facial nerve is achieved in 2 steps: the user draws the centerline of the nerve by clicking points on the CT slices (Fig. 2); a panoramic view of the nerve is reconstructed by unwrapping the previously defined centerline, where boundaries of the nerve are segmented manually. Special attention was taken to oversegment the nerve rather than undersegment it. Per animal, 3 groups of parallel trajectories were defined passing the facial nerve with distances of $1.0,0.5$, and $0.0 \mathrm{~mm}$. Finally, the plan including fiducial screw positions, facial nerve representation, and trajectory parameters are exported to be used intraoperatively by the robotic system.
FN Monitoring During Drilling

Each subject was immobilized on the OR table using an invasive pin-type head clamp. The robot system was mounted so that its end-effector could reach the intended drill position. An optical tracking reference was fixed on the mastoid bone within the workspace of the 3D optical tracking system. Two subdermal bipolar EMG electrodes were inserted into the Orbicularis Oris, and Orbicularis Oculi or Auricularis muscles. Electrodes for stimulation-return and EMG ground were placed at the base of the neck (Subjects 1 and 2) or centrally along the nasal bone (Subjects 3 and 4).

To begin the drilling phase, the temporal bone region was rigidly registered with the preoperative plan. The tunnel to the cochlea was then drilled in 2 phases by the robot system. In Phase 1, a shallow centering hole was created in the mastoid surface using a short spiral drill $(\varnothing 2 \mathrm{~mm})$. In the second phase, a stainless-steel spiral drill $(\varnothing 1.6 \times 35 \mathrm{~mm}$ length, $60-16035$, Stryker, Switzerland) with the shaft modified to be compatible with the Medtronic hand piece was used to drill the remaining tunnel with a speed of 5,000 rpm and feed forward rate of $0.25 \mathrm{mms}^{-1}$. These parameters were chosen to minimize the production of heat in the tissue, which may elicit a nerve response. Although specific temperature measurements were not performed, the drill was continuously irrigated, thus reducing the likelihood of nerve stimulation because of thermal effects. During this phase, the stimulation current pattern was swept among 3 different intensities as previously described. Peak EMG signals were extracted and plotted relative to the drill position in the navigation software (Fig. 3). The procedure was repeated for each planned trajectory, and the animal was euthanatized after completion of the experiment.

\section{Postoperative Analysis}

The actual positions of each drilled trajectory were used in the postoperative correlation of distance to $\mathrm{FN}$ and $\mathrm{EMG}$ response. 

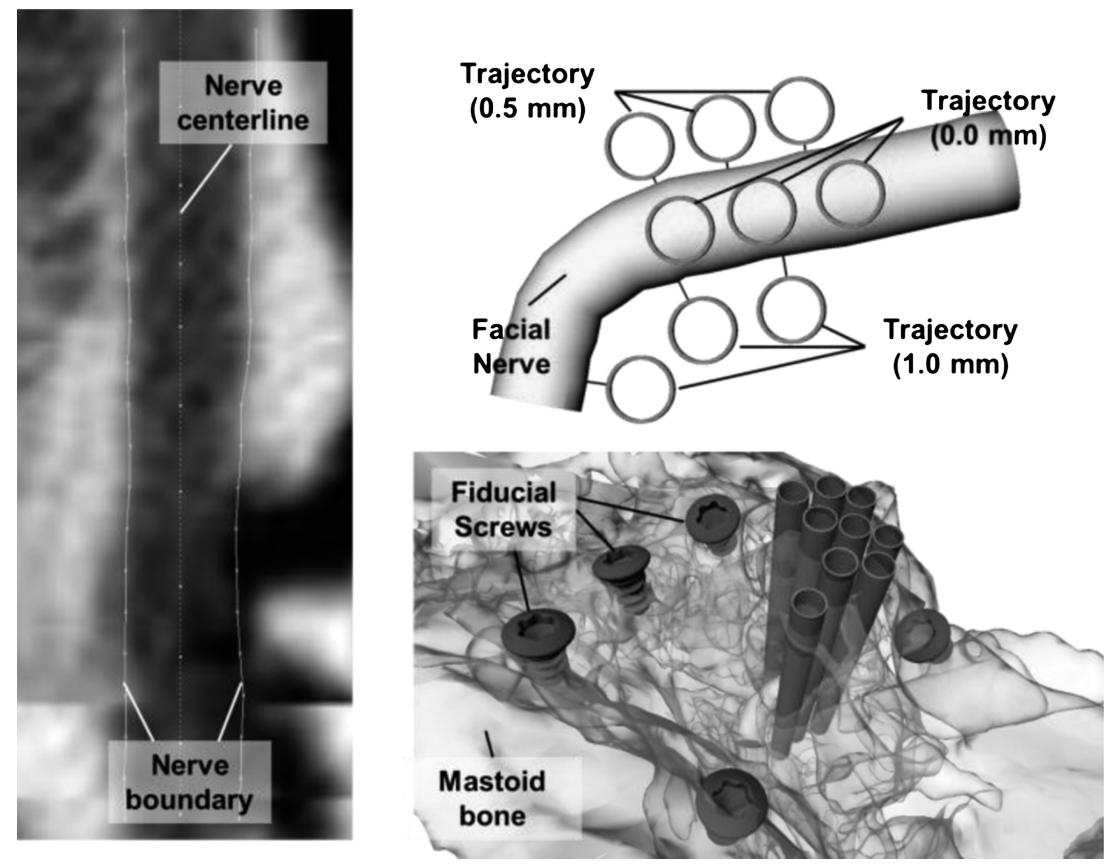

FIG. 2. The facial nerve boundaries are defined in a panoramic view reconstructed along the preselected nerve centerline (left). Thereafter, 3 groups of 3 trajectories with lateral distance to the surface of the facial nerve of $1,0.5$, and 0 mm were planned (top right). A 3D view of the fiducial screws, mastoid bone, facial nerve, and planned trajectories is shown in the final plan (bottom right).

This was accomplished by inserting $\varnothing 1.6 \mathrm{~mm}$ titanium wires in each tunnel (for increased contrast) before scanning the mastoid region using cone beam computed tomography (ProMax 3D Max, Planmeca, Finland) with an isometric voxel size of $0.15 \mathrm{~mm}^{3}$. Subsequently, corresponding preoperative and postoperative image data sets were coregistered using a mutual information algorithm in the commercial software Amira (Visage Imaging, Germany), as seen in Figure 4. This effectively transforms the postoperative data into the preoperative coordinate system for further comparison with the preoperative plan. The centerline of each titanium wire (representing the drilled hole) was determined in 2 steps. Voxel intensities higher than $2500 \mathrm{HU}$ were segmented in the image data using region growing and manual segmentation where necessary. The centerline (DCL) of each drilled hole was then calculated as the axis of the selected voxels using linear principle component analysis (Matlab, MathWorks, USA).

The recorded EMG signals were preprocessed using a median filter (medfilt1, Matlab, USA) to remove unwanted spikes above $2 \mathrm{KHz}$. The spikes were identified to be periodic interference coming from the active optical markers of the robot system. The filter was set with a $0.5-\mathrm{ms}$ running window. After removing the spikes, the EMG data were visually inspected to verify that the physiologic responses were not distorted by the filter.

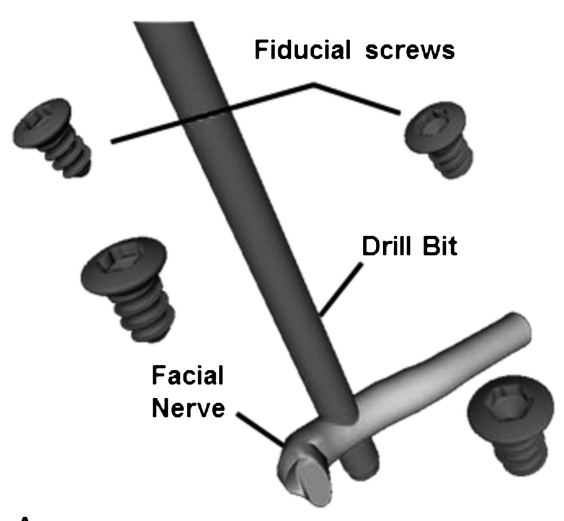

A

FIG. 3. $A, 3 \mathrm{D}$ view with the drill bit pose relative to the fiducial screws and the nerve (yellow) at the end of a drilled trajectory (Subject 4). $B$, The drill position (green line) is plotted relative to the centerline of the facial nerve in a 2D slice of the mastoid aligned with the drill trajectory. $C$, The extracted EMG peaks (response) from each channel as displayed during the drilling phase (bottom right corner). In this case, a sharp increase in peak EMG was observed during impact of the drill with the nerve. 


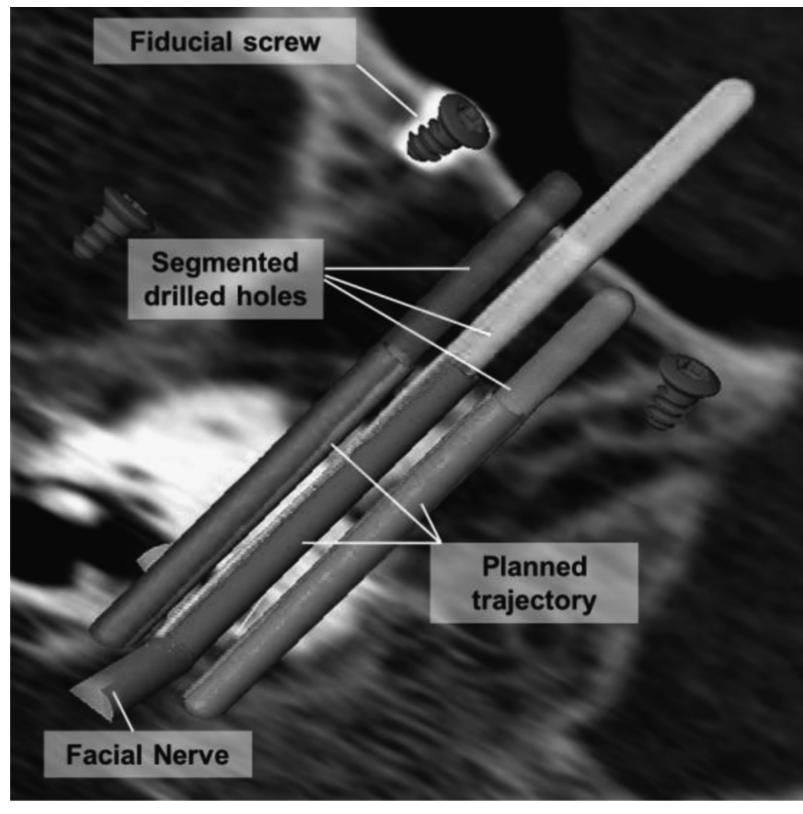

FIG. 4. 3D representation of the postoperative segmented wires superimposed over the preoperative planned trajectories (Subject 1 and Trajectories 1,2, and 3 in study results). The surface points of each wire were exported to Matlab for postoperative analysis.

The drill tip position relative to the EMG signal in the temporal domain was calibrated by finding the exact time point when the axial drilling force exceeded $1 \mathrm{~N}$, indicating contact was made with the temporal bone surface. Finally, the recorded EMG signals were mapped to a position along the drill trajectory using the calibrated robot position data. A stimulus response
(SR) was defined as an increase in the EMG peak value above a threshold 5 times the accumulated mean value of the signal. This value was chosen retrospectively as it was empirically observed that it corresponds to a detection of the nerve at distances between 0 and $3 \mathrm{~mm}$ for most of the analyzed cases. In practice, this results in an absolute threshold of 20 to $40 \mu \mathrm{V}$.

For further analysis, a coordinate system was defined using the drill centerline (DCL) and the lateral distance (LD) from the DCL to the FN center $\left(\mathrm{FN}_{\mathrm{c}}\right)$, as seen in Figure 5. The origin of the local coordinate system $(\mathrm{O})$ is defined as the intersection of DCL and LD. Two different distances between the FN and the drill bit were defined. The axial distance (AD) is defined as the drill position relative to the local origin O. Closest distance (CD) was defined as the shortest distance between drill bit and FN surfaces for each point along DCL. This terminology is summarized in Table 1. The stimulus response and the closest distance from the drill bit to the FN are analyzed using the defined coordinate system.

\section{Special Case}

In 1 animal, the response achieved with the drill system was compared with the response with a normal monopolar probe in 3 trajectories planned to pass through the FN. This was performed by stopping the robot $0.5 \mathrm{~mm}$ before the drill made contact with the fallopian canal. Thereafter, the drill was removed, and a monopolar probe (8225101, Medtronic, USA) was manually inserted to the end of the tunnel and EMG recordings were made. Following this intermediate step, the trajectories were drilled to the final depth as in all other cases.

\section{RESULTS}

To our knowledge, this work represents the first time a stereotactic system combining a high-precision image-guided

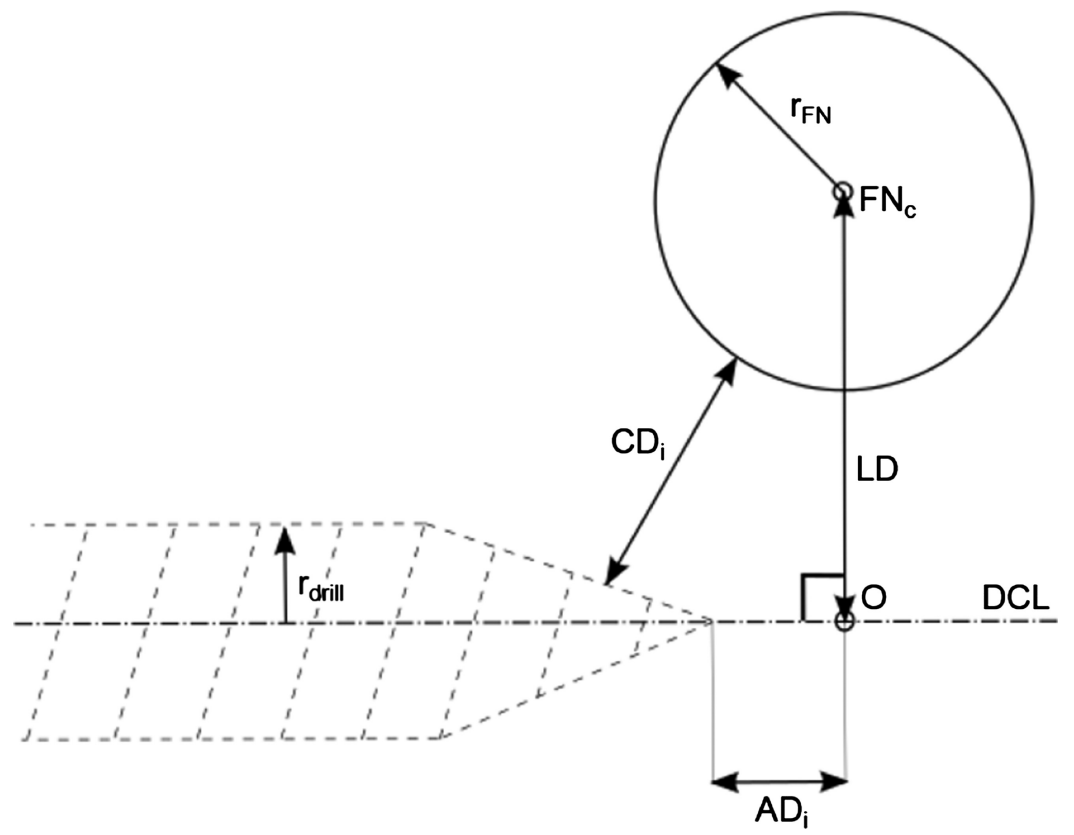

FIG. 5. Sketch representing the geometry used to define the EMG mapping coordinate system. The origin of the coordinate system (O) is the point on the drill center line $(\mathrm{DCL})$ with the shortest lateral distance to the center of the $\mathrm{FN}\left(\mathrm{FN}_{\mathrm{C}}\right) \cdot \mathrm{CD}_{\mathrm{i}}$ is the momentary closest distance between the drill bit and nerve at any point along the trajectory. 
TABLE 1. Briefing of the terminology used to define the electromyography mapping geometry

\begin{tabular}{ll}
\hline Abbreviation & \multicolumn{1}{c}{ Description } \\
\hline $\mathrm{DCL}$ & Calibrated drill centerline \\
$\mathrm{FN}$ & Facial nerve center \\
$\mathrm{LD}$ & Lateral distance from DCL to $\mathrm{FN}$ \\
$\mathrm{O}$ & Origin: Intersection of DCL and LD \\
$\mathrm{r}_{\mathrm{FN}}$ & Radius of facial nerve in plane $\mathrm{LD} \perp \mathrm{DCL}$ \\
$\mathrm{r}_{\mathrm{drill}}$ & Radius of drill bit \\
$\mathrm{CD}_{\mathrm{i}}$ & Closest distance from the drill bit surface to the \\
$\mathrm{AD}_{\mathrm{i}}$ & FN surface (3D measurement) \\
& Axial distance from drill tip to the origin of the \\
\hline
\end{tabular}

robotic tool and an intraoperative continuous NM system was used to measure EMG responses and correlate them with distances from the drill bit to the facial nerve as the drill bit was advancing through the mastoid bone. Using the described workflow, these measurements were performed in 4 animals resulting in 27 drilled trajectories. Some of the attempts had to be discarded from further analysis because of technical malfunctions. Subject 2 (6 trajectories) was discarded because of poor placement of the stimulation return and EMG ground electrodes during the experiment, which resulted in a high level of noise (common mode and base line artifacts) in the EMG signals. The base of the neck was concluded to be nonideal for the location of these electrodes because of the presence of fatty tissue and difficulty in finding a robust placement. Furthermore, in Subject 3, the postoperative analysis revealed that each of the 9 trajectories was shifted laterally with respect to the FN. Effectively, only 3 of the 9 drilled holes were within the originally planned lateral offset of 0 to $1 \mathrm{~mm}$ relative to the FN. The unusually large error in this subject was determined to be the result of an imaging artifact, perhaps caused by movement of the animal during the scan. In Subject 4, after observing no EMG response in the first 4 attempts, the electrode placement was changed from orbicularis oculi to the auricularis muscle as a stronger response was observed in this area. Thus, the first 4 attempts from this subject were excluded from the analysis. Finally, 11 of the original
27 drilling attempts, corresponding to 3 different subjects, were available for further analysis. The lateral, axial, and closest distances are recorded at the first stimulus response for each attempt in Table 2.

Although some EMG signals were detected in the final 200-ms window (Segment 3), these EMG events were accompanied by a response within Segment 2 (most likely electrically evoked). For simplicity, only the responses in Segment 2 (first 50-ms window) were reported in Table 2.

\section{Evaluation of CD at Different Stimulus Intensities}

As described previously, stimulation intensities of 1.5, 2.0 , and $2.5 \mathrm{~mA}$ were used in each of the drilling attempts. A scatter plot of the closest distance at first EMG response for each of these stimulation intensities is shown in Figure 6 . The $1.5-\mathrm{mA}$ intensity was not sufficient to elicit an EMG response except in 2 cases, in which the drill bit had already collided with the segmented boundary of the nerve $(\mathrm{CD}=0)$. Conversely, the 2.0 - and the $2.5-\mathrm{mA}$ intensities were more reliable in terms of eliciting an EMG response before contact with the nerve as indicated by CD greater than 0 (Fig. 6).

\section{Correlation of Stimulus Response to Distance}

In each panel of Figure 7, EMG response (solid blue line) and closest distance to the FN (dashed black line, $\mathrm{CD}_{\mathrm{i}}$ ) are plotted as a function of axial distance (AD). Additionally, a representation of the FN (yellow circle) with the appropriate LD and the drill bit position (gray polygon) at first stimulus response (SR) is depicted. The attempts from each subject with the largest and smallest lateral distances are shown in the left and right columns, respectively. Of the 3 different stimulation currents and the 2 available channels, the signal with the earliest response is plotted. In Panel 1, the closest distance (CD) at first SR is indicated with a line from the surface of the drill bit to the segmented boundary of the fallopian canal (FN boundary). The intersection between the LD and the DCL is depicted as the origin of the coordinate

TABLE 2. Compilation of the distances at first stimulus response for the 11 selected electromyography data sets

\begin{tabular}{|c|c|c|c|c|c|c|c|c|c|c|}
\hline \multirow[b]{3}{*}{ Subject } & \multirow[b]{3}{*}{ Trajectory } & \multirow{3}{*}{$\begin{array}{l}\mathrm{LD} \\
{[\mathrm{mm}]}\end{array}$} & \multirow{3}{*}{$\begin{array}{l}\mathrm{AD} \\
\text { at SR } \\
{[\mathrm{mm}]}\end{array}$} & \multicolumn{6}{|c|}{$\mathrm{CD}$ at first SR $[\mathrm{mm}]$} & \multirow{3}{*}{$\begin{array}{c}\text { FN penetrated } \\
(\mathrm{Y} / \mathrm{N})\end{array}$} \\
\hline & & & & \multicolumn{2}{|c|}{$1.5 \mathrm{~mA}$} & \multicolumn{2}{|c|}{$2.0 \mathrm{~mA}$} & \multicolumn{2}{|c|}{$2.5 \mathrm{~mA}$} & \\
\hline & & & & $\mathrm{CH} 1$ & $\mathrm{CH} 2$ & $\overline{\mathrm{CH}} 1$ & $\mathrm{CH} 2$ & $\mathrm{CH} 1$ & $\mathrm{CH} 2$ & \\
\hline \multirow[t]{3}{*}{1} & 1 & 2.5 & 1.2 & NR & NR & NR & 1.4 & 1.8 & 1.9 & $\mathrm{~N}$ \\
\hline & 2 & 2.2 & 0.6 & NR & NR & 1.0 & 1.0 & 1.0 & 1.0 & $\mathrm{~N}$ \\
\hline & 3 & 0.1 & 1.4 & NR & NR & NR & NR & NR & 0.4 & $\mathrm{~N}$ \\
\hline \multirow[t]{3}{*}{3} & 4 & 1.6 & 3.2 & NR & 2.1 & 0.1 & 0.0 & 0.0 & 0.0 & $\mathrm{Y}$ \\
\hline & 5 & 0.9 & 0.4 & NR & NR & 0.0 & 0.0 & 0.0 & NR & $\mathrm{Y}$ \\
\hline & 6 & 0.9 & 0.1 & NR & NR & NR & 0.0 & NR & 0.0 & $\mathrm{Y}$ \\
\hline \multirow[t]{5}{*}{4} & 7 & 1.7 & 0.2 & NR & 0.0 & NR & 0.0 & NR & 0.5 & $\mathrm{Y}$ \\
\hline & 8 & 1.5 & NA & NR & NR & NR & NR & NR & NR & Y \\
\hline & 9 & 0.4 & 0.1 & NR & NR & 0.0 & 0.0 & NR & 0.0 & $\mathrm{Y}$ \\
\hline & 10 & 0.3 & 0.4 & NR & 0.0 & NR & 0.0 & 0.0 & 0.0 & $\mathrm{Y}$ \\
\hline & 11 & 0.1 & NA & NR & NR & NR & NR & NR & NR & $\mathrm{Y}$ \\
\hline
\end{tabular}

NA indicates not applicable; NR, no response. 


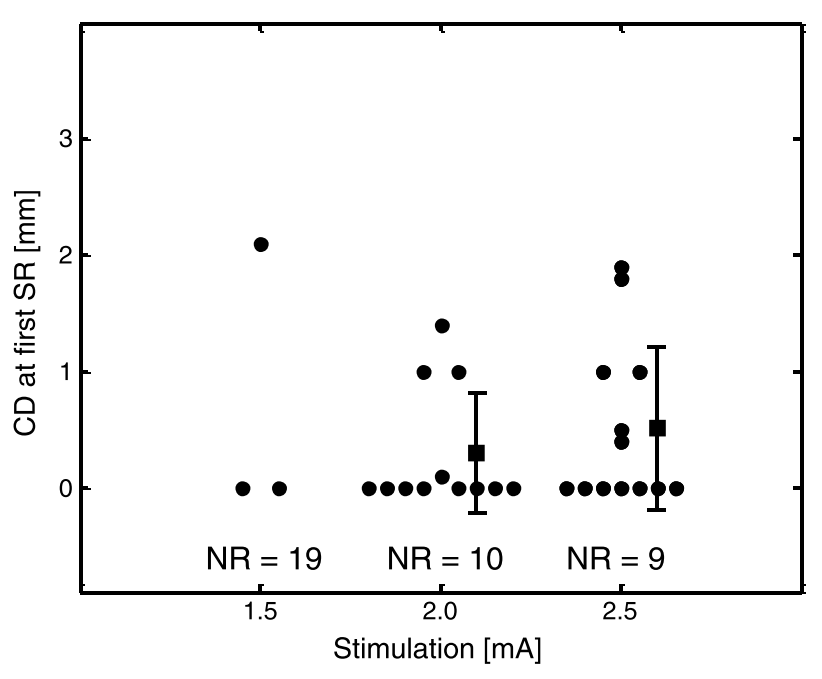

FIG. 6. The closest distance (CD) where the first stimulation response was measured for the 3 applied stimulation intensities is represented ( 11 drilled trajectories, 3 stimulation intensities and 2 channels per attempt). These results indicate that the 1.5-mA level is insufficient to elicit early warnings when approaching the FN. Conversely, the 2.5-mA level tended to be more reliable in terms of an early warning. Cases with no response are indicated as NR.

system $(\mathrm{O})$. This case represents a relatively large $\mathrm{LD}$ of $2.5 \mathrm{~mm}$.

An early response was defined as an EMG signal detected before the minimum closest distance $\left(\mathrm{CD}_{\text {min }}\right)$ is reached. Using this criterion, the lateral distance was compared between early and late responses for all 11 trials. To observe the trend of the data, each LD was represented in a scatter plot according to the category of early and late response as shown in Figure 8. Interestingly, trajectories with a larger LD tend to produce a response earlier than those with a shorter LD. Because of the limited number of samples in this study, a statistical test was not deemed appropriate.

In summary, only 2 of the 6 cases where a penetration of the facial nerve occurred, was an EMG response detected before collision with the segmented nerve boundary (Trajectories 4 and 7). Additionally, in 2 cases where collision with the FN occurred, no EMG response was detected even after contact was made (Trajectories 8 and 11). Only 1 case (Trajectory 3 ) exhibited an early detection with a small LD.

\section{Special Case}

No EMG response was measured using the stimulating drill in each of the 3 trajectories stopped $0.5 \mathrm{~mm}$ before reaching the FN (Trajectories 9-11). In contrast, signals between 75 and $85 \mu \mathrm{V}$ were recorded with the normal monopolar probe inserted to the end of the drilled tunnel.

\section{DISCUSSION}

For the first time, to our knowledge, a neuromonitoring (NM) enabled drill has been integrated with an imageguided surgical robot for DCA surgery and tested in a live animal study. Furthermore, a method to map EMG responses to distances from the drilling tool to the facial nerve was proposed and evaluated in 4 subjects.

Several limitations became apparent during the course of the study. First, the distance measurements to the FN are all based on image data where an idealized nerve is represented by a segmentation of the fallopian canal. Thus, the actual position of the FN is uncertain because of errors in the segmentation itself and the fact that the exact location of the nerve within this canal is also not completely known. The uncertainty of the nerve position can be addressed 2 fold. First, a more accurate representation of the fallopian canal through more precise segmentation will enable a more accurate model of the facial nerve. Furthermore, a micro-scale analysis of the nerve within the fallopian canal using micro-CT or histologic measurements could further refine the morphologic model of the nerve.

A second limitation of this work is the lack of a verification of the effectiveness of the electrode placement. This problem was made evident in Subject 4 where a repositioning of the electrodes resulted in a greatly improved EMG signal-to-noise ratio. A second method of stimulating muscle activity (next to NM integrated drill) would be beneficial in terms of assessing proper EMG electrode placement. This could be accomplished with a baseline stimulation through a needle electrode in the vicinity of the superficial branch of the FN innervating the orbicularis oris and could serve as a positive control ensuring effective EMG electrode placement. An additional electrode placed in the auricularis could be beneficial in the sheep model (in contrast to the human) as a strong signal was noted in this muscle during several experiments.

Although an anesthetic protocol designed to retain muscle activity was followed, an objective measurement of the actual anesthetic depth was not performed. The results of the special case, however, suggest that current shunting from the sides of the drill lead to poor sensitivity rather than a lack of muscle activity caused by the anesthetic depth. This statement is supported by the ability of the monopolar probe to elicit a response, whereas the stimulating drill did not. The main difference between the 2 tools is the presence of an insulating layer up to the tip of the monopolar probe and no insulation on the drill. The extent of irrigation and presence of fluid within the cavity can also have a detrimental effect. Kartush et al. (25) showed that the presence of liquids like cerebral spinal fluid could shunt current away from nerves. It was shown that this effect could be largely diminished by using electrodes insulated flush with the tip.

Initial test results indicate that reliable detection of the facial nerve is not possible using the current system. In fact, the analysis of the effect of lateral distance shows that a drill path heading directly toward the FN is less likely to elicit an early response than a more lateral path. These results contrast previously published findings where the same NM enabled drill system was used to detect the FN during schwannoma removal (17). In that work, the FN was detected before penetration of the fallopian canal 


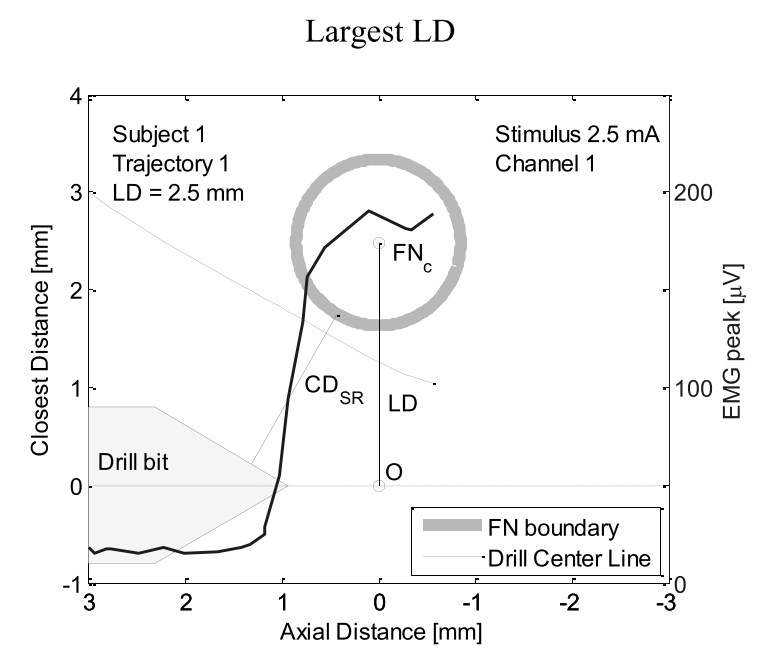

Subject 1
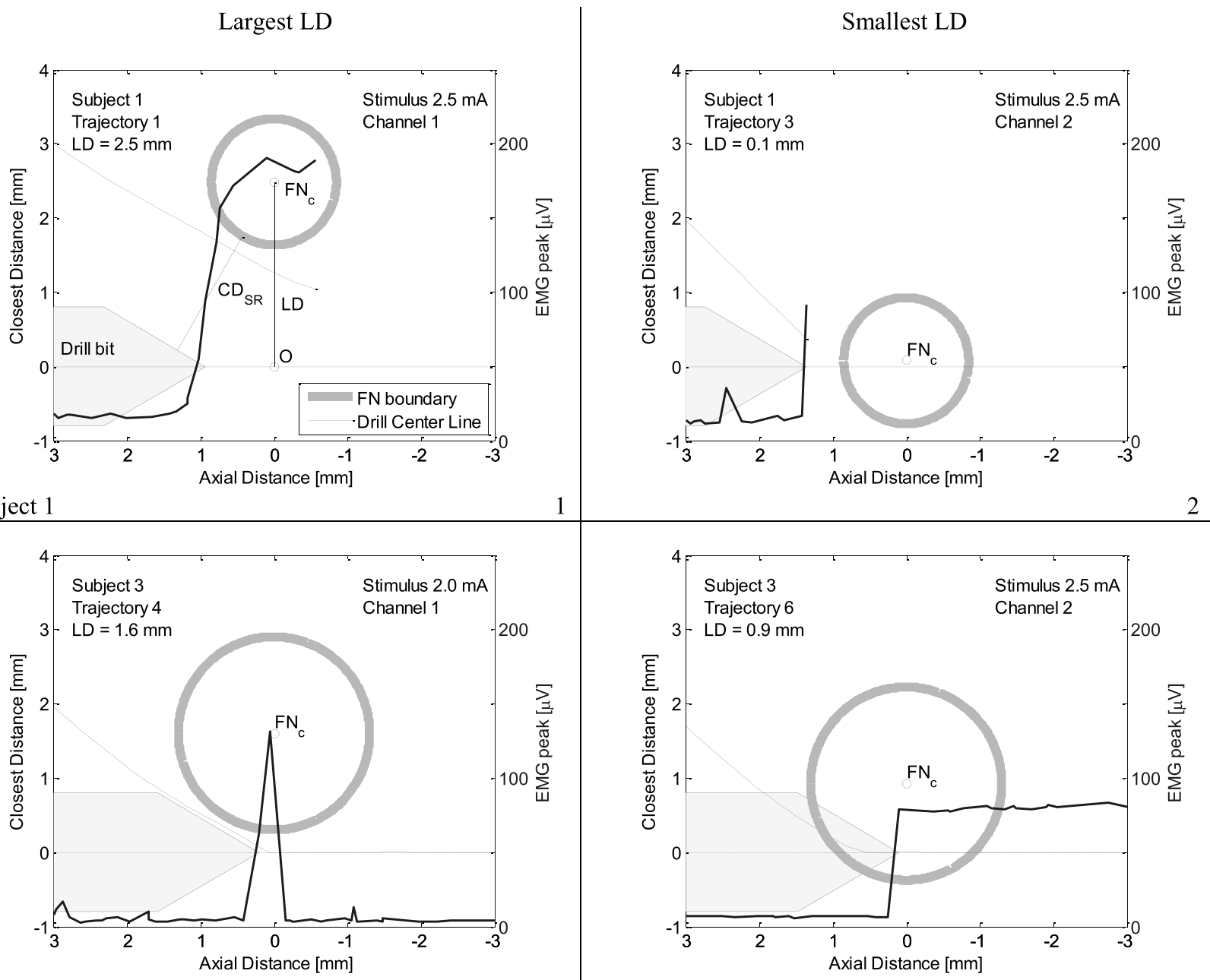

Subject 3

3
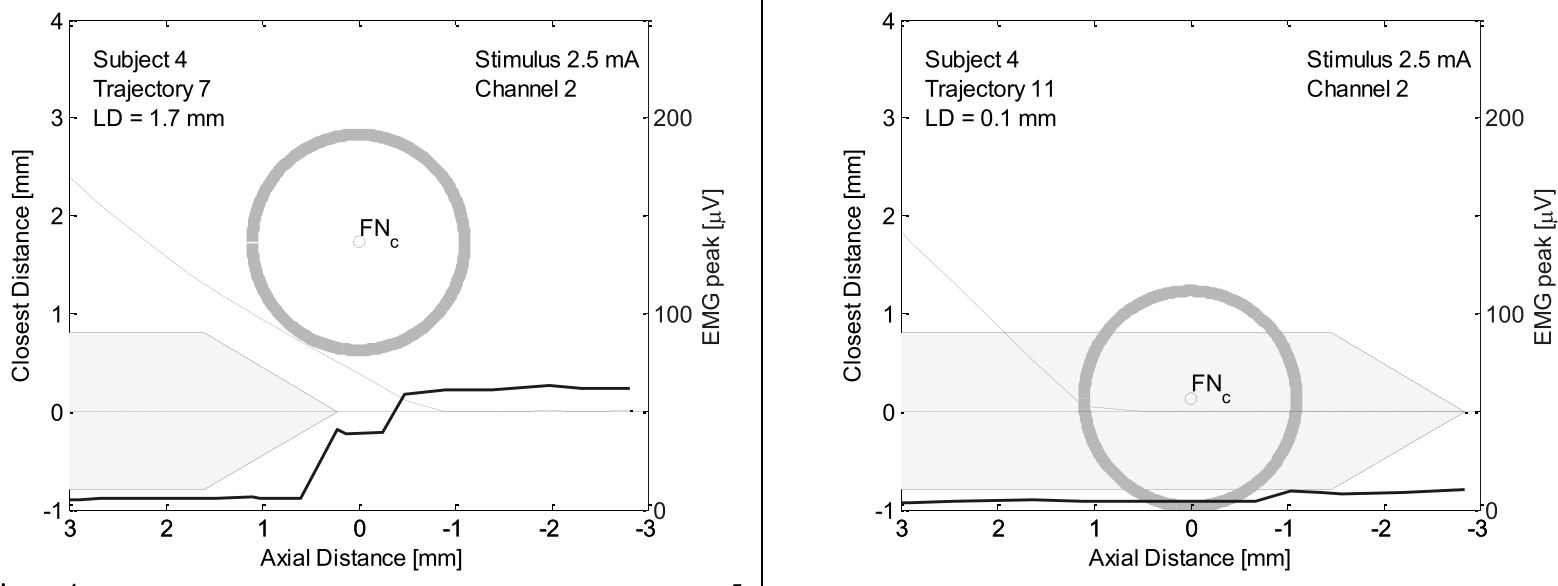

Subject 4

5

FIG. 7. Per subject, 2 different plots are depicted: the trajectory with the largest lateral distance (LD; left) and the attempt with the shortest LD (right). In each panel, the drill bit (gray polygon), the facial nerve boundary (yellow circle), the closest distance as a function of axial distance $\left(C D_{i}\right.$, black dashed line), the EMG peak signal (solid blue line), and the drill center line (DCL, black dot-dash line) are represented at the position of first stimulus response. In Panel 1, the closest distance at first stimulus response (CD $\left.{ }_{S R}\right)$ and LD are explicitly depicted for clarity.

Otology \& Neurotology, Vol. 35, No. 3, 2014 


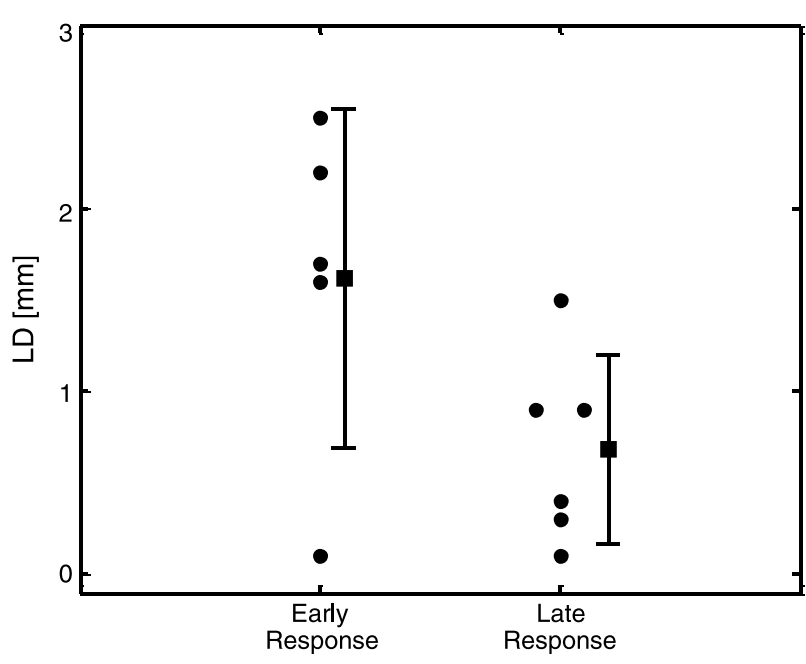

FIG. 8. Early responses are classified as a stimulus response elicited before the minimum closest distance $\left(C D_{\min }\right)$ is reached. Accordingly, detected EMG signals achieved after $C D_{\min }$ is reached were classified as late responses. A point representing the LD for each case is plotted according to this classification. The mean and standard deviation of the data are also depicted (error bars). The data show that cases with larger LDs tended to elicit an EMG response earlier than those with shorter LDs.

in 13 of 15 cases. There are, however, fundamental differences in the 2 methods. Bernardeschi et al. used a stimulating bur to mill out an open cavity in the mastoid bone with only a small portion of the bur in contact with the bone at any given time. This scenario mimics a monopolar probe wherein the shaft is insulated from the surrounding tissue, and only the tip may conduct the stimulus current (resulting in a controlled stimulation pattern).

In our approach, an uncoated stainless steel drill bit was used to create a small tunnel $(\varnothing 1.6 \mathrm{~mm})$. Thus, the stimulation current could "leak" into the tissue along the entire length of the drill bit. This effectively reduces the current density at the tip of the drill bit compared with the bur described previously. We believe that this altered stimulation pattern is the main cause of the observation that drill paths passing safely by the nerve seemed to be more likely to trigger an EMG response than those paths wherein a collision occurred. This hypothesis is corroborated by the fact that higher stimulation intensities $(2.0-2.5 \mathrm{~mA})$ were necessary to elicit a response in comparison with normal clinical use (0.8-1.2 $\mathrm{mA})$.

The original objective of the work was to determine the feasibility of using the drill integrated NM system to predict the proximity of the $\mathrm{FN}$ relative to the drill tip during robotic DCA. However, because of the deficiencies described previously, the detection of the FN (using the reported configuration) is not sensitive nor specific enough to be used as a warning for the surgeon during the robot-assisted procedure. The significantly different electrical stimulation pattern resulting from a noninsulated drill shaft within a tunnel means that the results of this study cannot be directly compared with results in an open mastoidectomy setting. To overcome this limitation, a more focused stimulation pattern must be realized. This can be accomplished with a drill designed specifically for this purpose, providing a conductive path only at desired locations. Investigations on such a tool are underway and will be tested in a future animal study. Finally, the results also indicate that more sophisticated patient-specific modeling of the electrical field may be necessary to improve the prediction of the distance from the tool tip to the FN.

Acknowledgments: The authors thank the VetSuisse faculty of the University of Bern, with Johann Lang, Claudia Spadavecchia, Franck Forterre, Anne Staudacher, Michael Stoffel, Helene Rohrbach, and Daniela Casoni for the support. The authors also thank Christian Weisstanner and Nadja Feusi with the Radiology team at Inselspital Bern. The authors also thank Josef Götte for the signal conditioning and Jürg Hüsler for the statistical representation of the data. The authors also thank Dawei Chen and Lilibeth Salas for the functional help with the logistics and the analysis of data.

\section{REFERENCES}

1. Häusler R. Cochlear implantation without mastoidectomy: the pericanal electrode insertion technique. Acta Otolaryngol 2002;122: 715-9.

2. Kronenberg J, Baumgartner W, Migirov L, Dagan T, Hildesheimer M. The suprameatal approach: an alternative surgical approach to cochlear implantation. Otol Neurotol 2004;25:41-4; discussion 44-45.

3. Labadie RF, Chodhury P, Cetinkaya E, et al. Minimally invasive, image-guided, facial-recess approach to the middle ear: demonstration of the concept of percutaneous cochlear access in vitro. Otol Neurotol 2005;26:557-62.

4. Schipper J, Aschendorff A, Arapakis I, et al. Navigation as a quality management tool in cochlear implant surgery. J Laryngol Otol 2004;118:764-70.

5. Labadie RF, Noble JH, Dawant BM, Balachandran R, Majdani O, Fitzpatrick M. Clinical validation of percutaneous cochlear implant surgery: initial report. Laryngoscope 2008;118:1031-9.

6. Wanna GB, Balachandran R, Majdani O, Mitchell J, Labadie RF. Percutaneous access to the petrous apex in vitro using customized micro-stereotactic frames based on image-guided surgical technology. Acta Otolaryngol 2009:1-6.

7. Majdani O, Rau TS, Baron S, et al. A robot-guided minimally invasive approach for cochlear implant surgery: preliminary results of a temporal bone study. Int J Comp Assist Radiol Surg 2009;4: 475-86.

8. Baron S, Eilers H, Munske B, et al. Percutaneous inner-ear access via an image-guided industrial robot system. Proc Inst Mech Eng H 2010;224:633-49.

9. Delgado TE, Bucheit WA, Rosenholtz HR, Chrissian S. Intraoperative monitoring of facial muscle evoked responses obtained by intracranial stimulation of the facial nerve: a more accurate technique for facial nerve dissection. Neurosurgery 1979;4:418-21.

10. Silverstein H, Smouha E, Jones R. Routine identification of the facial nerve using electrical stimulation during otological and neurotological surgery. Laryngoscope 1988;98:726-30.

11. Olds MJ, Rowan PT, Isaacson JE, Silverstein H. Facial nerve monitoring among graduates of the Ear Research Foundation. Am J Otol 1997; 18:507-11.

12. Sobottka SB, Schackert G, May SA, Wiegleb M, Reiss G. Intraoperative facial nerve monitoring (IFNM) predicts facial nerve outcome after resection of vestibular schwannoma. Acta Neurochir (Wien) 1998;140:235-42; discussion 242-3.

13. Hopkins C, Khemani S, Terry RM, Golding-Wood D. How we do it: nerve monitoring in ENT surgery: current UK practice. Clin Otolaryngol 2005;30:195-8. 
14. Wilson L, Lin E, Lalwani A. Cost-effectiveness of intraoperative facial nerve monitoring in middle ear or mastoid surgery. Laryngoscope 2003;113:1736-45.

15. Jackler RK, Selesnick SH. Indications for cranial nerve monitoring during otologic and neurotologic surgery. Am J Otol 1994;15: 611-3.

16. Leonetti JP, Matz GJ, Smith PG, Beck DL. Facial nerve monitoring in otologic surgery: clinical indications and intraoperative technique. Annal Otol Rhinol Laryngol 1990;99:911-8.

17. Bernardeschi D, Meskine N, Otaibi NAL, et al. Continuous facial nerve stimulating burr for otologic surgeries. Otology Neurotol 2011;32:1347-51.

18. Bell $\mathrm{B}$, Williamson $\mathrm{T}$, Gerber $\mathrm{N}$, et al. In vitro accuracy evaluation of image-guided robot system for direct cochlear access. Otol Neurotol 2013;34:1284-90.

19. Bell B, Stieger C, Gerber N, et al. A self-developed and constructed robot for minimally invasive cochlear implantation. Acta Otolaryngol 2012;132:355-60.
20. Gerber N, Gavaghan K, Bell B, et al. High accuracy patient-toimage registration for the facilitation of image guided robotic microsurgery on the head. IEEE Transact Biomed Eng 2013;60: 960-8.

21. Seibel VA, Lavinsky L, Irion K. CT-Scan sheep and human inner ear morphometric comparison. Br J Otorhinolaryngol 2006;72: 370-6.

22. Seibel VAA, Lavinsky L, De Oliveira JAP. Morphometric study of the external and middle ear anatomy in sheep: a possible model for ear experiments. Clin Anat 2006;19:503-9.

23. Soares HB, Lavinsky L. Histology of sheep temporal bone. $B r J$ Otorhinolaryngol 2011;77:285-92.

24. Gerber N, Bell B, Gavaghan K, Weisstanner C, Caversaccio M, Weber S. "Surgical planning tool for robotically assisted hearing aid implantation." Int J Comp Assist Radiol Surg (in press).

25. Kartush JM, Niparko JK, Bledsoe SC, Graham MD, Kemink JL. Intraoperative facial nerve monitoring: a comparison of stimulating electrodes. Laryngoscope 1985;95:1536-40. 